\title{
Borehole deformation under a stressed state and in situ stress measurement
}

\begin{abstract}
CY Wang State Key Laboratory of Geomechanics and Geotechnical Engineering, Institute of Rock and Soil
\end{abstract} Mechanics, Chinese Academy of Sciences, China

ZQ Han State Key Laboratory of Geomechanics and Geotechnical Engineering, Institute of Rock and Soil Mechanics, Chinese Academy of Sciences, China

YT Wang State Key Laboratory of Geomechanics and Geotechnical Engineering, Institute of Rock and Soil Mechanics, Chinese Academy of Sciences, China

JC Wang State Key Laboratory of Geomechanics and Geotechnical Engineering, Institute of Rock and Soil Mechanics, Chinese Academy of Sciences, China

\begin{abstract}
In engineering practice, in situ stress measurement is usually done using methods such as hydraulic fracturing and stress relief. Despite their advantages, these methods also have shortcomings including difficulty in determining stress directions, complexity of testing process, and limits in applicable borehole depth. In this study, we set out to investigate borehole morphology under stress based on theories of elastic mechanics, and propose an in situ stress measurement approach based on borehole deformation. We theoretically prove a circular hole plane under stress will be elliptical in shape, and derive the equations depicting the relationship between the elliptical parameters and the stresses. We developed a probe microscopy-based borehole measurement method, and performed laboratory simulation and system calibration tests to verify its viability and accuracy. This approach may serve as a new method for in situ stress measurement.
\end{abstract}

Keywords: elastic behaviour, in situ stress measurement, borehole geometric shape, microscopic displacement measurement

\section{Introduction}

In situ stress is the stress naturally present in the crust without the disturbance of engineering influence (Cai 1995). As the root cause of deformation and damage in underground and surface excavations, accurate data on in situ stress are important for understanding the rock mass mechanical properties and analysing pit wall stability. This is also required for engineering design and decision-making in excavation for geotechnical projects. Its importance only stands to increase with our exploration of the deeper crust, and new theories and techniques must be developed for its measurement.

At present, in situ stress is commonly measured by the hydraulic fracturing and stress relief methods (Qiao \& Cai 1995). The former is relatively unaffected by local factors, and especially has a high success rate on deeper measurements, but still has difficulty in determining the direction of the main stress. The latter allows for reliable and highly accurate measurements of stresses and their directions, yet is limited by complexity of the process, which involves elaborate drilling control throughout. Its application in deep boreholes is also impeded by the difficulty of setting up cables for sensors (Yao 2012). In summary, advancements are still needed in areas including the determination of stress directions, measurements of lower depths, reducing complexity of the process, and generally realising the in situ stress measurements for deep and ultra-deep boreholes in complex environments.

In this study, we employ elasticity theory to investigate the elliptical morphology of the borehole section under stress, establish the relationship between stress and its geometric parameters, and develop an 
approach that overcomes the limitations of existing methods. Our approach can provide easier and more reliable measurement of the strengths and directions of stresses, useful for in situ stress measurements in deep and ultra-deep boreholes.

\section{The circular hole under stress, and its elliptical parameters}

\subsection{Shape of the circular hole under stress}

Figure 1 depicts a hypothetical circular hole in an infinite, elastic thin plate, with the centre of the circle as the origin of coordinates (Wang \& Pan 1991; Pan 1989). The plate is under the influence of pull forces uniformly distributed along the $x$ and $y$-axes from infinitely far away. The displacement of any point $P$ on the wall of the hole can be determined by the following equation:

$$
\begin{aligned}
& u=u_{1}+u_{2}=\frac{a}{E}\left[\sigma_{1}+\sigma_{2}+2\left(\sigma_{1}-\sigma_{2}\right) \cos (2 \theta)\right] \\
& v=v_{1}+v_{2}=-\frac{2 a}{E}\left(\sigma_{1}-\sigma_{2}\right) \sin (2 \theta)
\end{aligned}
$$

where:

$u$ and $v$ are respectively the displacements of $P$ on the radial and tangential directions.

$\sigma_{1}$ and $\sigma_{2}$ are respectively the strengths of pulls along the $x$ and $y$-axes.

$\theta$ is the angle between the radial direction at $P$ and the positive $x$-axis.

$a$ is the radius of the circle.

$E$ is the elastic modulus of the plate.

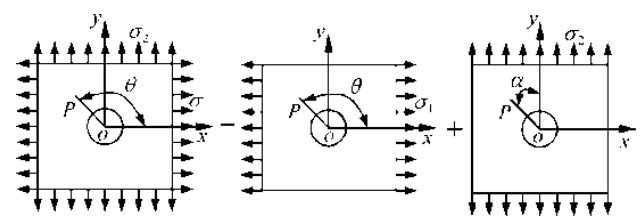

Figure 1 Schema of displacements on walls of the hole

As shown in Figure 2, suppose point $P$ is moved to point $P^{\prime}$ by the hole's deformation, with the coordinates $(x, y)$, then we have:

$$
\left.\begin{array}{l}
x=a \cos \theta+u \cos \theta-v \sin \theta \\
y=a \cos \theta+u \sin \theta+v \cos \theta
\end{array}\right\}
$$

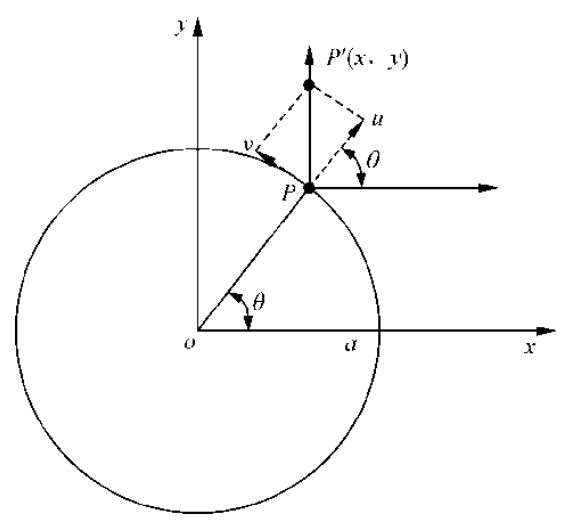

Figure 2 Deformation at any point on walls of the hole 
Substitute Equation (1) into Equation (2), and the following can be obtained after simplification:

$$
\left.\begin{array}{l}
x=a\left[1+\left(3 \sigma_{1}-\sigma_{2}\right) / E\right] \cos \theta \\
y=a\left[1+\left(3 \sigma_{2}-\sigma_{1}\right) / E\right] \sin \theta
\end{array}\right\}
$$

which satisfies the following:

$$
\frac{x^{2}}{\left[a\left(1+\left(3 \sigma_{1}-\sigma_{2}\right) / E\right)\right]^{2}}+\frac{y^{2}}{\left[a\left(1+\left(3 \sigma_{2}-\sigma_{1}\right) / E\right)\right]^{2}}=\cos ^{2} \theta+\sin ^{2} \theta=1
$$

and this is a standard elliptical equation. This proves the deformed shape of the circular hole under stress to be elliptical (Wang et al. 2016; Verrujit 1998).

Let $A$ and $B$ be respectively the semi-major and semi-minor axes of the ellipse, and we have:

$$
\left.\begin{array}{l}
\sigma_{1}=\frac{3 A+B-4 a}{8 a} E \\
\sigma_{2}=\frac{A+3 B-4 a}{8 a} E
\end{array}\right\}
$$

\subsection{Determining elliptical parameters}

Figure 3 establishes a Cartesian coordinate system, where the point 0 is the centre, the $x$-axis is the horizontal axis with its positive direction pointing left, and the $y$-axis is the vertical axis with its positive direction pointing up. For three points $P_{1}, P_{2}$ and $P_{3}$ on the ellipse, their respective centrally symmetric points are $P_{1}^{\prime}, P_{2}^{\prime}$ and $P_{3}^{\prime}$. Suppose the respective distances between them $\left|P_{1} P_{1}^{\prime}\right|,\left|P_{2} P_{2}^{\prime}\right|$ and $\left|P_{3} P_{3}^{\prime}\right|$ are $D_{1}, D_{2}$ and $D_{3}$, and the angles between the positive $x$-axis and the lines between them are respectively $\theta_{1}, \theta_{2}$ and $\theta_{3}$, and let $l_{i}=D_{i} / 2(i=1,2,3)$. The coordinates of $P_{1}, P_{2}$ and $P_{3}$ can then be determined:

$$
\left.\begin{array}{c}
x_{i}=l_{i} \cos \theta_{i} \\
y_{i}=l_{i} \sin \theta_{i}
\end{array}\right\}
$$

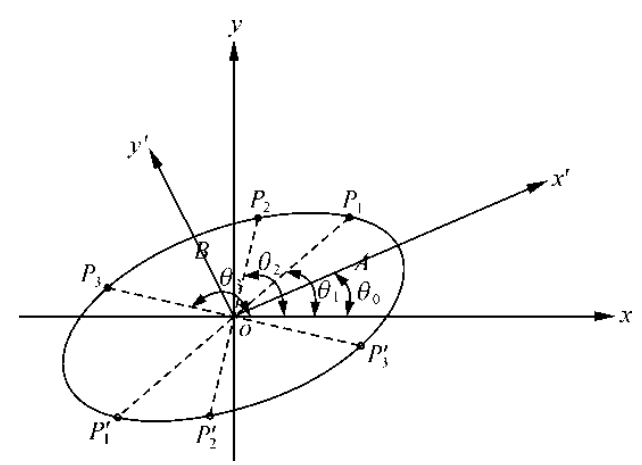

Figure 3 Principles for determining elliptical parameters

Suppose the angle between the ellipse's major axis and $x$-axis is $\theta_{0}$, and the $x^{\prime}$-axis of the ellipse-centred coordinate system $x^{\prime} o y^{\prime}$ overlaps with the major axis. The elliptical equation under coordinate system $x^{\prime} o y^{\prime}$ can be represented by:

$$
\frac{x^{\prime 2}}{A^{2}}+\frac{y^{\prime 2}}{B^{2}}=1
$$

where:

$A$ and $B$ are respectively the semi-major and semi-minor axes, with $A>B$. 
Under coordinate system $x^{\prime} o y^{\prime}$, the coordinates of $P_{1}, P_{2}$ and $P_{3}$ are:

$$
\begin{aligned}
& x_{i}^{\prime}=l_{i} \cos \left(\theta_{i}-\theta_{0}\right) \\
& y_{i}^{\prime}=l_{i} \sin \left(\theta_{i}-\theta_{0}\right) \quad(i=1,2,3)
\end{aligned}
$$

Substitute Equation (8) into Equation (7), and solve the simultaneous equations, resulting in:

$$
\begin{aligned}
& A^{2}=\frac{\left[\cos ^{2}\left(\theta_{1}-\theta_{0}\right)-\cos ^{2}\left(\theta_{2}-\theta_{0}\right)\right] D_{1}^{2} D_{2}^{2}}{4\left[D_{2}^{2} \sin ^{2}\left(\theta_{2}-\theta_{0}\right)-D_{1}^{2} \sin ^{2}\left(\theta_{1}-\theta_{0}\right)\right]} \\
& B^{2}=\frac{\left[\cos ^{2}\left(\theta_{1}-\theta_{0}\right)-\cos ^{2}\left(\theta_{2}-\theta_{0}\right)\right] D_{1}^{2} D_{2}^{2}}{4\left[D_{1}^{2} \cos ^{2}\left(\theta_{1}-\theta_{0}\right)-D_{2}^{2} \cos ^{2}\left(\theta_{2}-\theta_{0}\right)\right]}
\end{aligned}
$$

For the sake of simplicity, we let $\theta_{2}=\theta_{1}+45^{\circ}$ and $\theta_{3}=\theta_{1}+90^{\circ}$, and two solutions can be obtained:

$$
\theta_{0}=\theta_{1}+\frac{1}{2} \arctan \frac{D_{1}^{2} D_{2}^{2}-2 D_{1}^{2} D_{3}^{2}+D_{2}^{2} D_{3}^{2}}{2\left(D_{1}^{2} D_{2}^{2}-D_{2}^{2} D_{3}^{2}\right)}
$$

and

$$
\theta_{0}=\theta_{1}+\frac{1}{2}\left[\arctan \frac{D_{1}^{2} D_{2}{ }^{2}-2 D_{1}^{2} D_{3}{ }^{2}+D_{2}{ }^{2} D_{3}^{2}}{2\left(D_{1}^{2} D_{2}{ }^{2}-D_{2}^{2} D_{3}^{2}\right)}+180\right]
$$

With $A>B$, the two solutions can be substituted into Equation (9) to determine their respective $A$ and $B$ values. The actual solution can then be found via comparison.

\section{Measurement of elliptical morphology}

Based on the determination process for elliptical parameters in the previous section, we only need to measure the 'diameter' of the borehole along three different directions to determine its elliptical shape. This allows us to develop an approach that enables simultaneous measurement of borehole diameter along three or more directions using optical probe microscopy.

\subsection{Probe}

For this study, we used symmetrically-placed rigid measurement elements, or probes, as shown in Figure 4(a). At least three pairs of probes are symmetrically located within each horizontal plane, as shown in Figure 4(b). The probes are kept in close contact with borehole walls by elastic elements (such as springs). Each probe has a pin facing against the walls, and pointing into a tiny area in the centre. The deviations from borehole 'diameter' are determined by a combination of directional data from the probes and optical data from the pins.

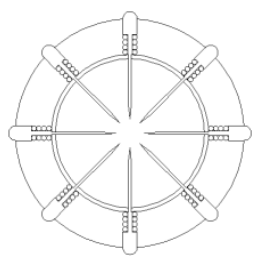

(a)

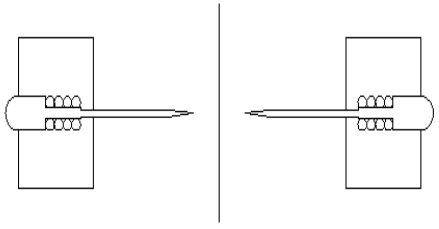

(b)

Figure 4 (a) Measuring pins with symmetrical arrangement; (b) details of measuring pin 


\subsection{Optical microscopy imaging}

The tiny area of each pin point is the target of the optical imaging process. The small size of the target area necessitates the introduction of microscopy imaging, implemented with a lens of a small vertical angle of view (i.e. having a relatively long focal length), and a compatible charge coupled device (CCD) image sensor.

\subsection{Direction measuring}

The optical image alone cannot represent the actual horizontal direction the measurement is made on. This necessitates a direction finder component. Figure 5 shows a schematic of the full measurement system, complete with measurement components, microscopic lens, CCD image sensor, and direction compass. The direction finder can be a planar or three-dimensional e-compass. All components are located on the same central axis.

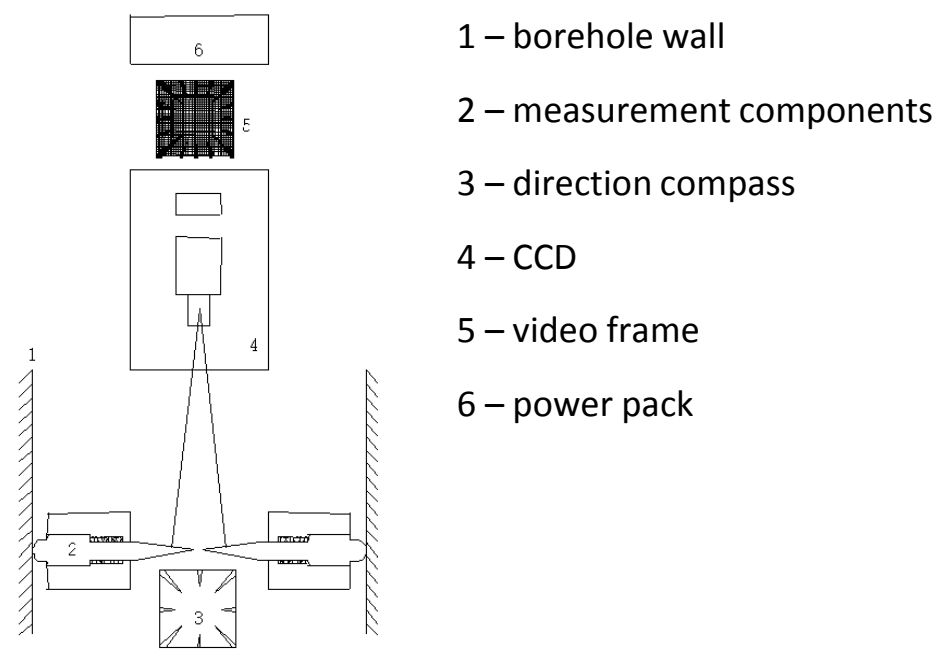

Figure 5 Schematic of the measurement system

\subsection{Establishing the base circle}

When a pin is first placed in an initial location on the target plane, this location can be calibrated to serve as the basis for measuring the actual borehole dimensions. For this purpose, we designate a standard base circle, which usually has the smallest diameter possible for the probe system. When all probes are placed along the circle, the locations of the pins are taken as their initial locations. Image records of the initial locations are made, and used to establish the coordinate system of the base circle.

\subsection{Calculation}

Under the base coordinate system, let $P(t)$ be the point where a pin is located at moment $t$, and $Q(t)$ be the point where its symmetric pin is located at moment $t$, and $D_{0}$ be the diameter of the base circle. Initially the points have coordinates $P_{0}(X, Y)$ and $Q_{0}(-X,-Y)$, and the distance between the points is $L_{0}=\left|P_{0} Q_{0}\right|$. Therefore $L_{0}=D_{0}$.

At moment $t$, the distance between the two pin points $L(t)$ is:

$$
L(t)=|P(t) Q(t)|=\left|P(t) P_{0}\right|+\left|P_{0} Q_{0}\right|+\left|Q_{0} Q(t)\right|
$$

Because $\left|\mathrm{P}(t) \mathrm{P}_{0}\right|$ and $\left|\mathrm{Q}_{0} \mathrm{Q}(t)\right|$ together represent the deviation from the base circle's diameter, let $k$ be the accuracy factor of the system (detailed calculation in the next section), the corresponding actual change to the diameter $\Delta D(t)$ is:

$$
\Delta D(t)=k\left(\left|P(t) P_{0}\right|+\left|Q_{0} Q(t)\right|\right)
$$


While $\left|P_{0} Q_{0}\right|$ corresponds to the actual diameter of the circle, i.e. $\left|P_{0} Q_{0}\right|=D_{0}$, therefore the actual diameter of the borehole is $D(t)$ :

$$
D(t)=D_{0}+\Delta D(t)
$$

The direction of this diameter can be determined using the e-compass system.

\section{$4 \quad$ Laboratory experiment}

\subsection{Image calibration test}

The changing coordinates of pin points in the image reflect the deformation of the pins. Calibration is required to determine the relationship between the coordinates and the deformation. The calibration was done using a GWB-200JA extensometer, shown in Figure 6, with accuracy up to $0.0002 \mathrm{~mm}$.

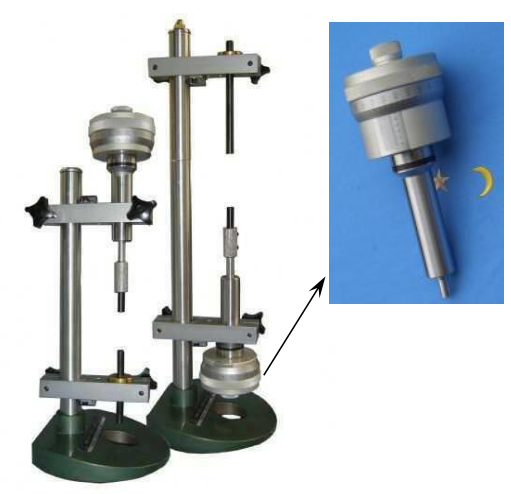

\section{Figure 6 Calibration instrument}

The calibration involves the following stages:

1. The system is fixed to the calibration device. The pin is coaxial with the device's measurement shaft, and its movement is synchronous with the device.

2. The device's measurement shaft is used to push the pin in steps of $0.001 \mathrm{~mm}$, and the full scale result of each step is recorded.

3. The previous stage is repeated until all pins are tested.

4. Images of pins from each step are recorded to calculate their coordinates.

5. The initial locations are determined, and used as basis to determine the relationship between the pin's displacement on image coordinates, and the actual distance moved by the device. Thus the accuracy factor is obtained, representing the ellipse's radial deformation corresponding to a unit pixel, and its unit is $\mu \mathrm{m} /$ pixel.

Suppose the calibration device causes a standard displacement of $S_{d}$ on the pin. System software is used to calculate the image coordinates of the pin, obtaining its image displacement, which is $I_{d}$ (unit: pixel). Thus the system's accuracy factor $k$ (the actual displacement represented by a unit pixel) is:

$$
k=\frac{S_{d}}{I_{d}}
$$

The results of the calibration experiment are shown in Figure 7. 

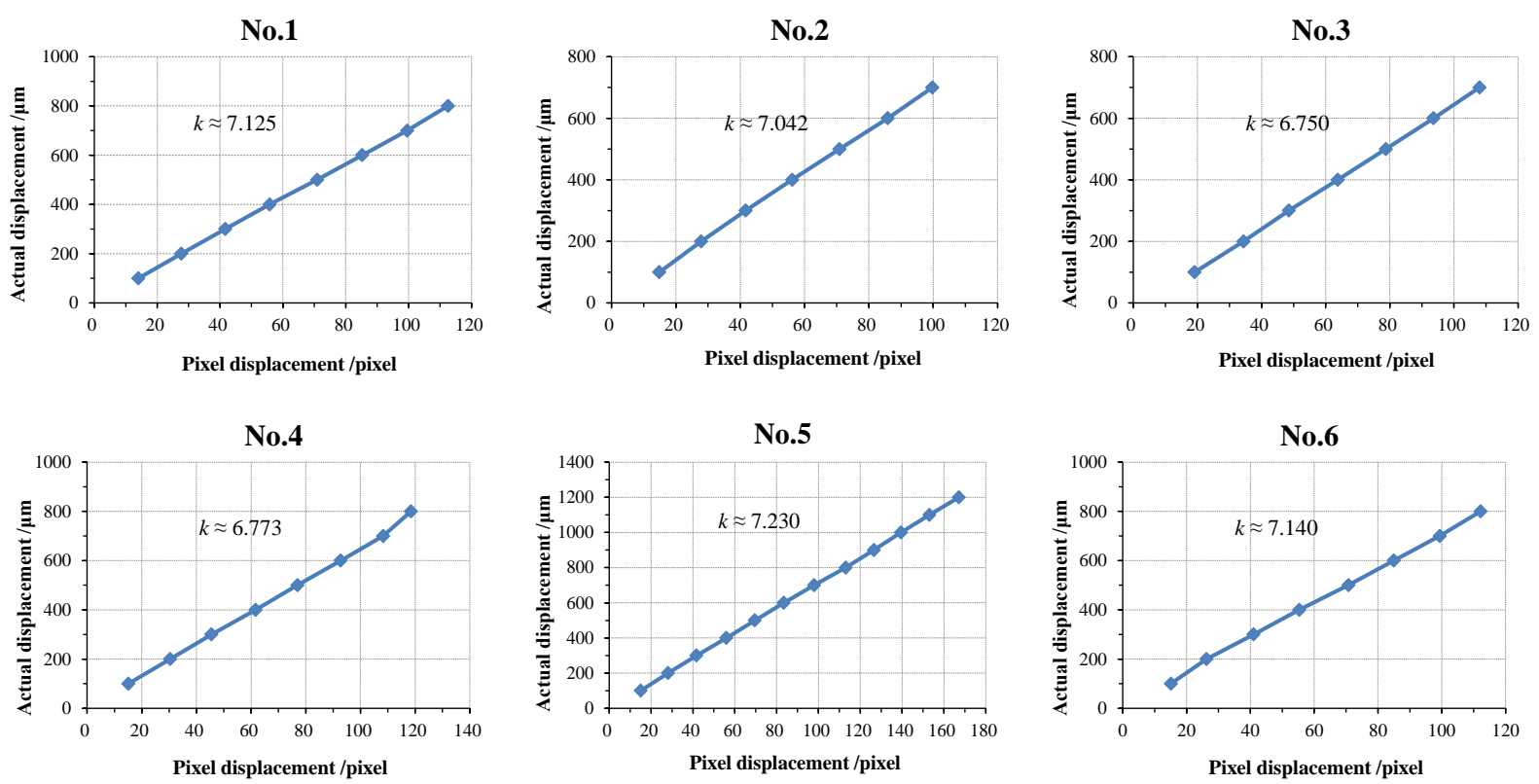

Figure 7 Results of the calibration test

According to tests, the six pins respectively have the following accuracy factors: $7.125,7.042,6.750,6.773$, 7.230, and 7.140, with an average of:

$$
\bar{k}=\frac{\sum_{i=1}^{6} k_{i}}{6}=7.01
$$

and a standard deviation of:

$$
S=\sqrt{\sum_{i=1}^{6}\left(k_{i}-\bar{k}\right)^{2}}=0.45
$$

Since the pins do not differ significantly in accuracy, the system's accuracy factor can be taken to be their average, i.e. each pixel corresponds to $7.01 \mu \mathrm{m}$ of actual displacement.

\subsection{Borehole morphology measurement and stress determination}

In order to verify the method's viability, load tests were performed on two cubic specimens with boreholes. The test was performed with a rock mechanics test (RMT) device, with a highest load of $500 \mathrm{kN}$. Specimen 1 consisted of a piece of highly isotropic nylon material with elastic modulus $E_{1}=1.4 \mathrm{GPa}$; Specimen 2 was a shale specimen, which elastic modulus had been determined by test to be $E_{2}=15 \mathrm{GPa}$. Both specimens had a borehole with a diameter of $40 \mathrm{~mm}$ in the centre. For each test, the probe microscopy system was placed inside the borehole as shown in Figure 8, and uniaxial pressure was added to the specimen.

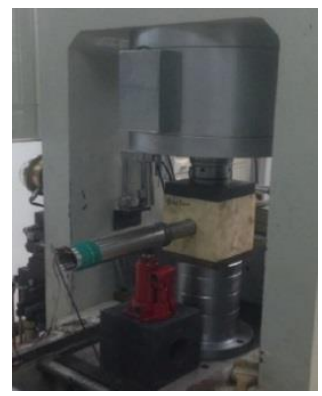

Figure 8 Borehole deformation test under loading condition 


\subsubsection{Determining stress}

After each specimen was put in place, its load was increased in steps of $50 \mathrm{kN}$ until $250 \mathrm{kN}$ (for nylon) or $300 \mathrm{kN}$ (for shale). During the loading process, the probe system was used to detect the displacements of multiple points on the borehole walls. The results were recorded as real-time videos of the pins' changes. Afterwards, system software was used to analyse the borehole's deformation, using Equations (5), (8) and (9) to determine the stresses that cause the deformation. The results are shown in Figure 9. For the nylon specimen, the errors between the result and the actual load are within the 0.19-0.74 MPa range; for the shale specimen, the errors are in the 0.44-1.24 MPa range.

Nylon test block

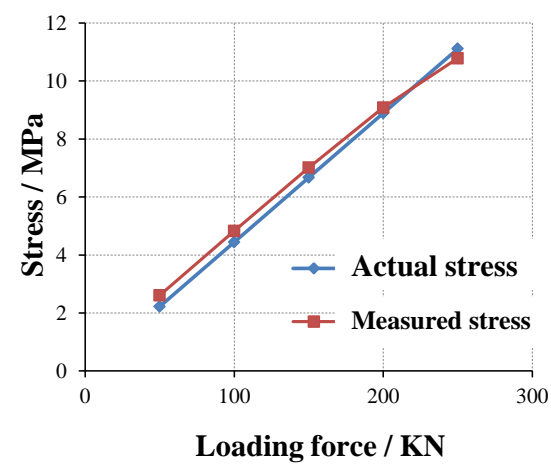

Shale test block

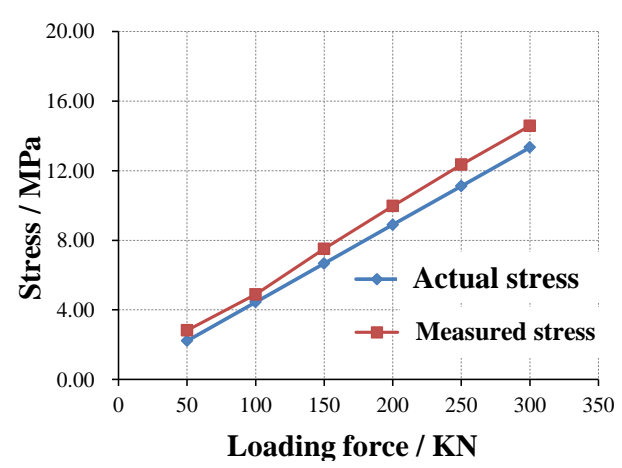

Figure 9 Comparison of actual stress and measured stress

Based on the test, the system can determine the in situ stress with accuracies above $86.7 \%$, with smaller errors from the nylon specimen than the shale specimen. From Figure 10, it can be seen that the nylon specimen's borehole deformation was visibly greater than that of the shale specimen, indicating this approach can obtain more accurate results from scenarios with higher stresses and deformations.
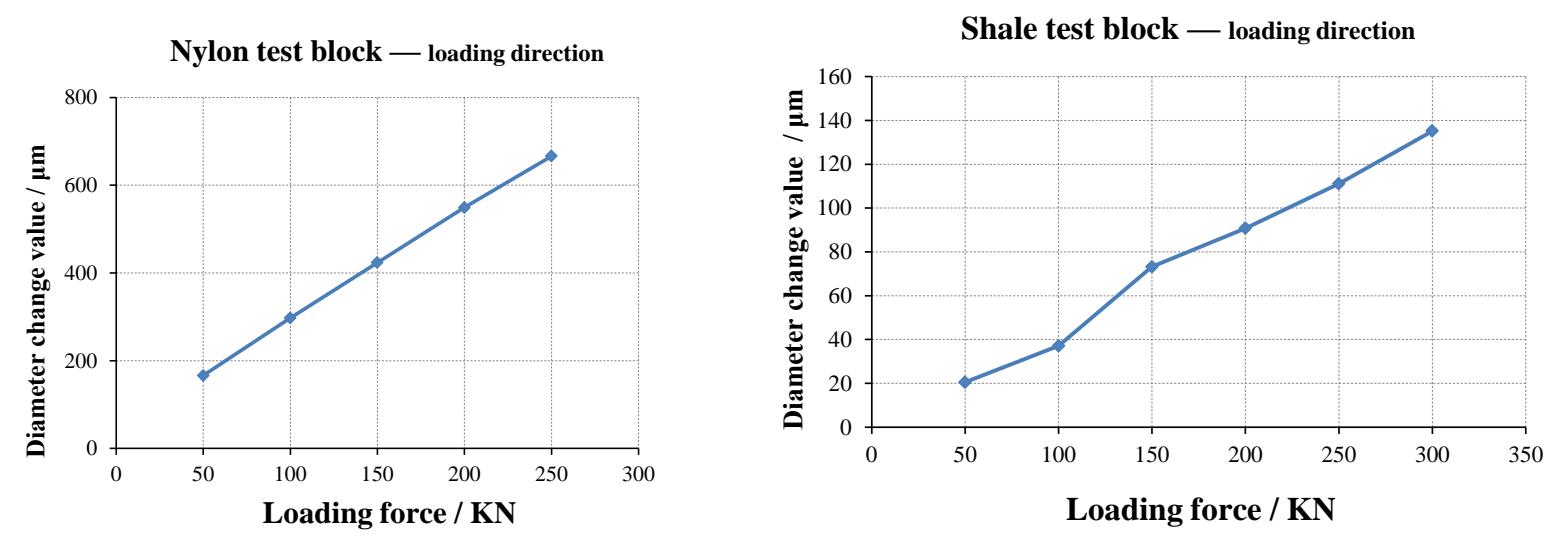

Figure 10 The variation of the ellipse short axis (loading direction) during the loading process

\subsubsection{Determining the direction of the maximum main stress}

Based on Equation (9), the direction of the main stress can be determined from the variations of three probe pairs, and the directions of one pair among the three. Notably it is also unrelated to the elastic modulus of the rock body, or other material parameters. This greatly simplifies the computation process. The results from the test are shown in Figure 11. It can be seen that the error is in the 1-3 degree range between the calculated and actual directions for the nylon specimen, and in the same range for the shale specimen. 

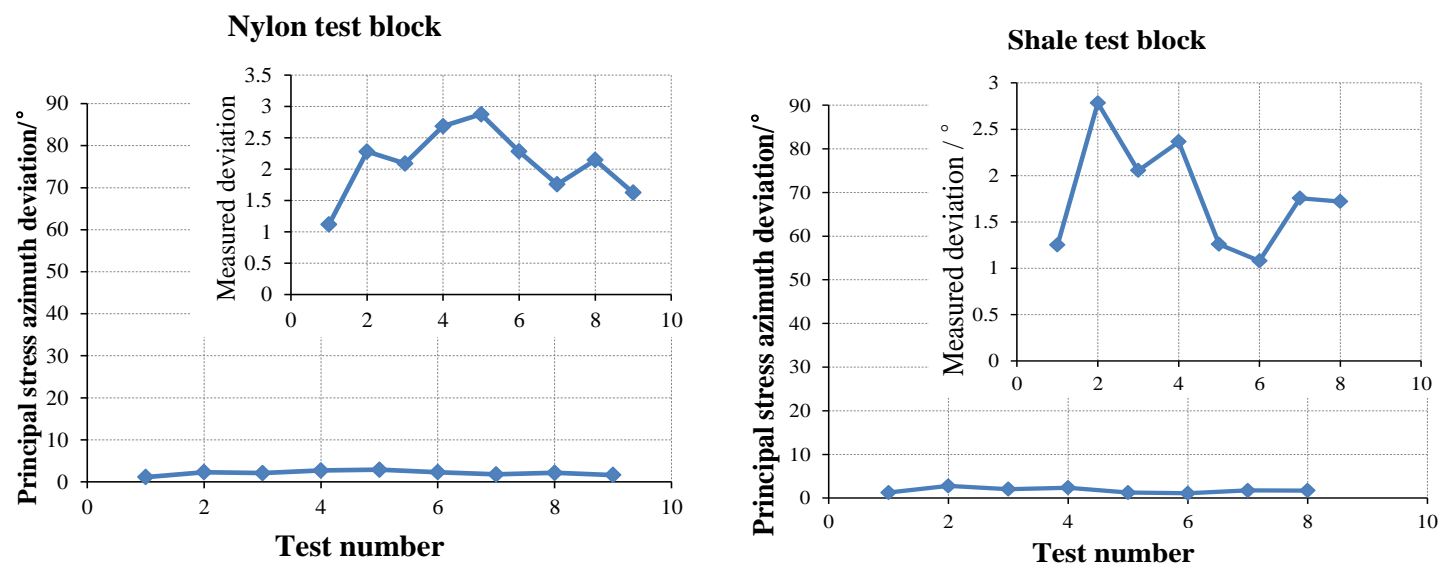

Figure 11 Measurement deviation of the direction of the major principal stress

It can be concluded that the direction of maximum main stress determined by this method has a low error, making it an accurate method that does not need additional information, i.e. material parameters of the measured rock body.

\section{$5 \quad$ Concluding remarks}

In this study, we build on theories of elastic mechanics to investigate the geometric shape of a circular hole after stress-induced deformation, and the determination of its elliptical parameters. This lays the foundation for an in situ stress testing method based on borehole morphology. A probe microscopy approach is proposed, and its viability and validity is verified by laboratory simulation test. This approach may serve as a new method for in situ stress measurements.

\section{Acknowledgement}

This study is subsidised by the Strategic Research Program of the Chinese Academy of Sciences (XDB10030200) and by the National Natural Science Foundation of China (No. 41402278 \& No. 41372317).

\section{References}

Cai, M 1995, The principle and technology of in situ stress measurement, Science Press, Beijing, pp. 68-90.

Pan, L 1989, 'Stress ellipsoid and stress ellipse', Bulletin of The Institute of Geomechanics, Chinese Academy of Geological Sciences, vol. 12, pp. 113-144.

Verruijt, A 1998, 'Deformation of an elastic half plane with a circular cavity', International Journal of Solids and Structures, vol. 35 , no. 21, pp. 2795-2804.

Qiao, L \& Cai, M 1995, 'New development of stress relief method for determination of in-situ stresses in a gold mine', Chinese Journal of Rock Mechanics and Engineering, vol. 14, no. 1, pp. 25-32.

Wang, C, Han, Z, Wang, J \& Wang, Y 2016, 'Study of borehole geometric shape features under plane stress state', Chinese Journal of Rock Mechanics and Engineering, vol. 35, supp. 1, pp. 2836-2842.

Wang, L \& Pan, L 1991, Crustal stress measurements and their application in engineering, Geological Publishing House, Beijing, pp. 1-31.

Yao, R, Yang, S \& Lu, Y 2012, 'Computing maximum and minimum horizontal stresses in in situ stress measurements', Chinese Journal of Geotechnical Engineering, vol. 34, no. 2, pp. 317-325. 
